\title{
Effect of zinc oxide and titanium dioxide nanoparticles on supported lipid bilayers
}

\begin{abstract}
Nanoparticles (NP) are broadly exploited in biomedical sciences in order to develop various methods of targeted drug delivery, novel biosensors and new therapeutic pathways. However, relatively little is known in the negotiation of NPs with complex biological environments. NP interaction with cell membranes can damage the cell membrane and cause toxicity; therefore, examining interactions between NPs and cell membranes is crucial to understanding NP toxicity mechanisms and the development of safe and nontoxic NP-based commercial and therapeutic applications. To gain a physical understanding of NP-membrane interactions, we used a simplified system built of well-defined synthetic lipid bilayers and NP. The interaction of nanoparticles (NPs) with supported lipid bilayers (SLB) can lead to structural modification of the SLB and affect the structure and dynamic of lipids. In this work $\mathrm{TiO}_{2}$ and $\mathrm{ZnO}$ nanoparticles were chosen because of wide applications and usage in industry of papers, inks, medicines, food products, cosmetics, toothpastes and skin care products and among others. Therefore, a better understanding of the interactions between NP and lipid membrane may help to better clarify the potential risk of NPs. The interaction between $\mathrm{ZnO}$ and $\mathrm{TiO}_{2}$ NPs and lipid membranes was studied by using scanning supercritical angle fluorescence microscopy. The biological response to elucidated changes in lipid membrane structure/characteristics under $\mathrm{ZnO}$ or $\mathrm{TiO}_{2} \mathrm{NP}$ influence was tested by fluorescence correlation spectroscopy. It was found the significant reduction of lipids diffusion mobility, which can be explained as a result of lipid- $\mathrm{ZnO}$ aggregates binding, depending on $\mathrm{ZnO}$ concentration. $\mathrm{The}^{\mathrm{TiO}_{2}}$ has a little effect on lipids diffusion mobility.
\end{abstract}

Volume 2 Issue 3 - 2015

\author{
Nora Grinceviciute, ' Dorinel Verdes, ${ }^{2}$ \\ Valentinas Snitka' \\ 'Department of Mathematics and Life Sciences, Kaunas \\ University of Technology, Lithuania \\ 2University of Zurich, Switzerland
}

\author{
Correspondence: Valentinas Snitka, Department of \\ Mathematics and Life Sciences, Research Centre for \\ Microsystems and Nanotechnology, Kaunas University of \\ Technology, Studentu 65, LT-51369, Kaunas, Lithuania, Tel 37037 \\ 45I588, Fax 3703745 I588 Email vsnitka@ktu.it
}

Received: April 30, 2015 | Published: May 25, 2015

Keywords: ZnO Nanoparticles, $\mathrm{TiO}_{2}$ Nanoparticles, Lipid membrane, Supercritical angle fluorescence

Abbreviations: NP, Nanoparticles; SLB, Supported Lipid Bilayers; BLMs, Bilayer Lipid Membranes; SAF, Supercritical Angle Fluorescence; FCS, Fluorescence Correlation Spectroscopy; PBS, Phosphate Buffered Saline; DLS, Dynamic Light-Scattering

\section{Introduction}

Lipid membranes are essential components of any living cell and therefore, bilayer lipid membranes (BLMs) are simplified planar models of cell membranes, commonly employed for both fundamental and applied studies. BLMs as a model system are of great importance for improving our understanding of cell interaction with environment, various disease mechanism and membrane properties for drug screening purposes. Important area of nanomedicine research is the interaction of nanoparticles with cell and a possible toxicity. ${ }^{1-5}$ Apart from their mechanical function as a cell wall, lipid membrane performs a function of a transfer mechanism for molecules diffusing through the membrane. As this process could distort the membrane structure, characterization tools that enable us to study the structure (ordering) of the membrane also provide information about the influence of NPs on the membranes. $\mathrm{ZnO}$ and $\mathrm{TiO}_{2}$ nanoparticles and their interaction with biological objects were investigated by several authors using different methods. ${ }^{6-10}$ however the mechanism of NP interaction with membrane is still an open question. Supercritical Angle Fluorescence Microscopy (SAF) is a technique that allows detection and characterisation of fluorescent species (proteins, biomolecules, pharmaceuticals, etc.) and their behaviour very close or even adsorbed or linked at surfaces.

The method is able to observe molecules at a distance of up to 100 nanometers from the surface even in the presence of high concentrations of fluorescent species around. ${ }^{11-12}$ In this work, we address two issues related to the interpretation of the fluorescence of phospholipid systems. While useful information can be retrieved from SAF microscopy, including the detection of very subtle effects, also the interpretation of the fluorescence correlation spectroscopy feature has not yet been settled, at least not in paperson NP's influence for lipid bilayer. Here, we specifically focus on the effect of different types and concentrations of NP's on lipid diffusion in supported lipid bilayers (SLBs). We used two different NPs: $\mathrm{ZnO}$ and $\mathrm{TiO}_{2}$ with 1 $\mathrm{mg} / \mathrm{ml}$ and $2.5 \mathrm{mg} / \mathrm{ml}$ concentrations. The NP's are attached to the supported lipid bilayer by ionic interactions between positively charged NP's suspension and negatively charged lipid. The diffusion properties are determined by fluorescence correlation spectroscopy (FCS). FCS used to determine diffusion coefficient of SLB's alone and with NP's, to demonstrate the NP influence on lipid bilayer structure and molecular dynamics.

\section{Materials and methods}

All reagents and solvents used in this study were analytical grade. $\mathrm{ZnO}$ nano-powder ZincOx10 was obtained from Nanogate AG, Titanium dioxide nano powder from $\mathrm{Na}$ Bond, phosphate buffered saline (PBS), Tris buffer, EDTA, $\mathrm{NaCl}, \mathrm{CaCl}_{2}, \mathrm{NaN}_{3}$ and 2-propanol from Sigma Aldrich. Lipids 1,2-dioleoyl-sn-glycero-3-phospho-L-serine (DOPS) and 1,2-dioleoyl-sn-glycero-3-phosphocholine (DOPC) were obtained from Sigma Aldrich and 1,2-dioleoyl-sn-glycero-3phosphoethanolamine (DOPE) from ATTO-TECH.

\section{Preparation of $\mathrm{ZnO}$ and $\mathrm{TiO}_{2}$ suspension}

Weighted $5 \mathrm{mg}$ of $\mathrm{ZnO} / \mathrm{TiO}_{2}$ powder was sonicated for $15 \mathrm{~min}$ at 20 ${ }^{C} \mathrm{C}$ in an ultrasonic bath along with $50 \mu \mathrm{l}$ of 2-propanol. Next, $5 \mathrm{ml}$ of PBS was added and the suspension $(1 \mathrm{mg} / \mathrm{ml})$ was sonicated for $1 \mathrm{~h}$ at 
$20^{\circ} \mathrm{C}$. For the higher concentration suspension preparation $(2,5 \mathrm{mg} / \mathrm{ml})$ $12,5 \mathrm{mg}$ of $\mathrm{ZnO} / \mathrm{TiO}_{2}$ powder was sonicated for $15 \mathrm{~min}$ at $20^{\circ} \mathrm{C}$ in an ultrasonic bath along with $125 \mu \mathrm{l}$ of 2-propanol and $5 \mathrm{ml}$ of PBS was added and the suspension was sonicated for $1 \mathrm{~h}$ at $20^{\circ} \mathrm{C}$.

\section{Substrate preparation}

As support for the lipid bilayers, glass cover slips were used. The pieces (ca. $2.5 \times 4 \mathrm{~cm}$ ) were cleaned in ethanol and sonicated for 15 min at $20^{\circ} \mathrm{C}$ in an ultrasonic bath. Before use, these substrates were stored in ethanol. Before use, substrates were rinsed several times with Milli-Q water, dried with nitrogen gas and affected with low pressure plasma (Electronic Diener) for 10 minutes. For the bilayer preparation the substrates were glued onto an aluminium sample cell.

\section{Supported lipid bilayers}

Supported lipid bilayers were prepared following a modified protocol of Richter et al. ${ }^{13}$ In short, (DOPS) and DOPC) and (DOPE) in chloroform were used as received and mixed at a ratio of 35:65:0, 000016. The lipid was dried overnight under vacuum to remove the solvent and then resuspended in Tris buffer $(149 \mathrm{mMNaCl}, 5 \mathrm{mM}$ $\mathrm{CaCl}_{2}, 10 \mathrm{mMTris}$ ) to a final concentration of $0.1 \mathrm{~g} / \mathrm{L}$. The resulting solution was extruded 27 times through a membrane (pore size $0.1 \mathrm{~m}$ ) to produce unilamellar vesicles. The vesicle solution was put directly in the measurement cell containing the glass substrate. There the solution was left for more than $1 \mathrm{~h}$ and after that it was replaced by the pure Tris buffer. Subsequently, this solution was exchanged again by $\mathrm{a} \mathrm{Ca}^{2+}$ free Tris buffer $(10 \mathrm{mMTris}, 133 \mathrm{mMNaCl})$ containing $5 \mathrm{mM}$ EDTA in order to remove the $\mathrm{Ca}^{2+}$ ions and finally by PBS. All buffers contained $0.05 \% \mathrm{NaN}_{3}$ andwere adjusted to $\mathrm{pH} 7.4$.

\section{SAF and FCS measurements}

Images of fluorescently labelled lipid membranes were recorded with a custom-made scanning microscope allowing detection of fluorescence and the supercritical angle fluorescence (SAF) images of an emitter simultaneously. The SAF channel detects only fluorophores in close proximity to the surface ( $\square 100-200 \mathrm{~nm}$ ) and efficiently rejects the fluorescence from the bulk solution using parabolic lenses as the decisive optical element. A detailed description of the optical setup is provided in the work. ${ }^{14}$ All measurements were conducted by passing buffered solutions of NP's over the lipid membrane through the flow cell at a constant pump rate of $250 \mu \mathrm{l} / \mathrm{min}(0.42 \mathrm{~mm} / \mathrm{s})$. This flow rate is clearly slow enough to have no effect on the supported lipid bilayer integrity. Raw scan images are presented as measured using appropriate linear scaling for the signal intensities. Note that the background subtraction in all images is based on a fixed intensity threshold. The shown images are selected representative images from time-lapse experiments which were conducted at varying time intervals, because the applied setup (non-commercial) does not contain the option of a software controlled time-lapse measurement.

Fluorescence Correlation Spectroscopy (FCS) is a method that was introduced and established as a very suitable approach to characterize membranes. ${ }^{14,15}$ A single-molecule method with the dramatically improved sensitivity provides sufficient statistical significance to also use it for general characterization of membranes, mainly through the diffusion properties of their constituents (diffusion coefficients, concentrations, molecular brightness, intra molecular dynamics and molecular interactions). ${ }^{16,17}$ FCS was employed by several authors to measure the lateral lipid mobility and to assess the impact of NP's on lipid dynamics in supported lipid membranes. ${ }^{18-22}$ A hardware correlator translates the photon arrival pulses into intensity fluctuations and calculates the correlation in real time. The correlation curves were acquired and fitted to analytical expressions. For an averaged correlation measurement, a separate correlation measurements of 100 seconds duration were taken and corresponding standard deviations for every point of the experimental curves were calculated from multiple experiments. The diffusion coefficient of the lipids in the supported lipid bilayer was determined from the experimentally obtained correlation time and the known waist of the confocal observation volume.

\section{Zeta potential measurements}

All measurements were made at a temperature of $25^{\circ} \mathrm{C}$ on a DelsaNanoC (Beckman Coulter) fitted with a Flow Cell coupled to Auto Titrator. For all measurements, a voltage of $60 \mathrm{~V}$ was applied across an electrode spacing of $16 \mathrm{~mm}$, the angle detection being of 15 . The solutions used for $\mathrm{pH}$ titration were $0.1 \mathrm{M} \mathrm{HCl}$ and $0.1 \mathrm{M} \mathrm{NaOH}$. The measurement for electrophoretic mobility was converted into zeta potential using Smoluchowski's formula. ${ }^{23-25}$ Atomic force microscopy measurements The Atomic Force Microscopy (AFM) investigations for the $\mathrm{ZnO}$ and $\mathrm{TiO}_{2}$ nanoparticles were done in the tapping mode (NT-MDT Inc.) using commercial silicon cantilevers NSG11 with a force constant of $5 \mathrm{Nm}-1$. The $\mathrm{ZnO}$ or $\mathrm{TiO}_{2}$ water solution $(1 \mathrm{mg} / 1 \mathrm{ml})$ was filtered by $200 \mathrm{~nm}$ polyethersulfone pore membrane (Chromafil PES-20/25, Macherey-Nagel) and placed onto the glass substrate (Carl Roth, $50 \times 24 \mathrm{~mm}, \# 1)$ and then dried and measured.

\section{Dynamic light scattering measurements}

Size characterization of the samples was made by dynamic lightscattering (DLS) measurements using the same DelsaNanoC fitted with a Size Cell, which uses a $4 \mathrm{~mW} \mathrm{He}-\mathrm{Ne}$ laser operating at a wavelength of $658 \mathrm{~nm}, 30 \mathrm{~mW}$ and a detection angle of 15 . Dispersions ( $1 \%$ Solution of $\mathrm{TiO}_{2}$ or $\mathrm{ZnO}$ in water) were made at $3.24 \mathrm{mg} / \mathrm{ml}$ in Nano-pure water with 16 minutes of sonication on ice bath according with NRCWE protocol used for similar analysis. Size characterization of the samples was made by DLS measurements using the same DelsaNanoC fitted with a Size glass cell, which uses a detection angle of $160^{\circ}$. Approximately $2 \mathrm{ml}$ of the suspension was introduced to a glass size cell using a plastic pipette tip.

\section{Transmission electron microscopy measurements}

The TEM analyses were performed using a JEOL JEM 2010F electron microscope operating at an accelerating voltage of $200 \mathrm{kV}$, with a point to point resolution of $0.19 \mathrm{~nm}$ (Joint Research Center, Ispra). The microscope was equipped with a Gatan electron energy loss imaging filter with an energy resolution equal to $0.7 \mathrm{eV}$ and a multiscan CCD $1 \mathrm{k} \mathrm{x} 1 \mathrm{k}$ CCD camera for image acquisition.

\section{Results and discussion}

\section{Characterization of nanoparticles}

AFM and TEM were used to determine the size of NPs. The grain analysis function was used and then the distribution of NPs size was built. It gives the size of $\mathrm{ZnO}$ of $20.2 \mathrm{~nm}$ (standard deviation (SD) $-9.47 \mathrm{~nm}$ ) and $\mathrm{TiO}_{2}$ gave the size value of $35.3 \mathrm{~nm}(\mathrm{SD}-9.20 \mathrm{~nm}$ ) (Figure 1). DLS was used by the instrument to determine the size distribution of particles by measuring dynamic fluctuations of light scattering intensity caused by the Brownian motion of the particle. This technique yields a hydrodynamic diameter that is calculated via the Stokes-Einstein equation from the aforementioned measurements. The measurement gives as result the average hydrodynamic diameter of the particles, the peak values in the hydrodynamic diameter (dh) distribution and the polydispersityindex (PdI) that describes the width 
of the particle size distribution (Figure 2a-2f). Also, zeta potential measurements were performed. The magnitude of the zeta potential indicates the degree of electrostatic repulsion between adjacent, similarly charged particles in dispersion. $\mathrm{ZnO}$ zeta potential variation with $\mathrm{pH} 7-8.5$, dispersions are stable and positively charged (with $\mathrm{pH} 7.4 \sim+3 \mathrm{mV}$ ). It is known, that low zeta potentials tend $\mathrm{ZnO}$ suspension to coagulate or flocculate. $\mathrm{In}_{\mathrm{TiO}}$ zeta potential variation with $\mathrm{pH}$ 5-9, dispersions are stable and surface is positive (with $\mathrm{pH} 7.4$ $\sim 33 \mathrm{mV}$ ). In this case, a high zeta potential will confer $\mathrm{TiO}_{2}$ stability, i.e., the suspension resists aggregation.

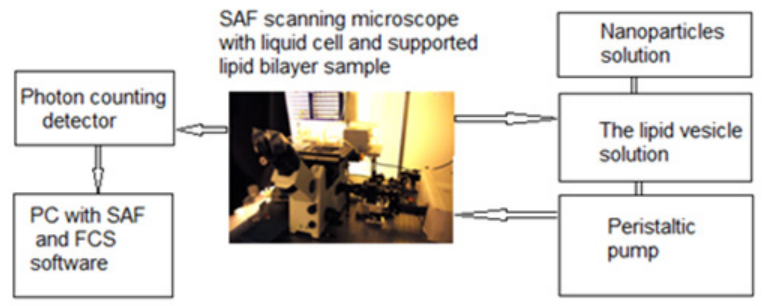

Figure I The experimental set-up for SAF imaging and FCS measurements.
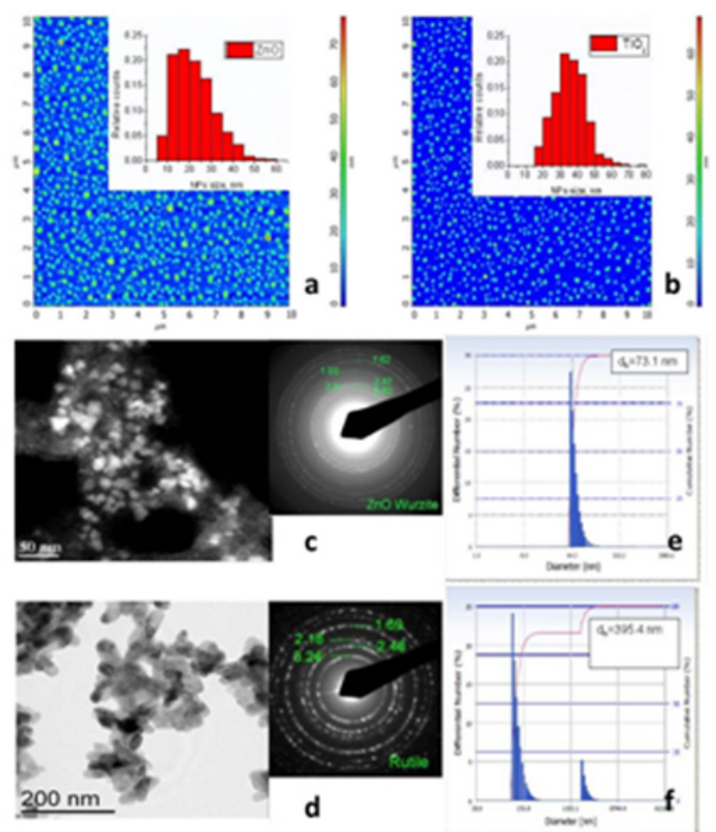

Figure 2 AFM measurements and characterization, TEM images and selected area electron diffraction pattern and DLS Differential number vs. Diameter for $1 \%$ solution in water of $\mathrm{ZnO}(\mathrm{a}, \mathrm{c}, \mathrm{e})$ and $\mathrm{TiO} 2$ (b, d, f) nanoparticles, respectively.

\section{ZnO and TiO2 NP's interaction with SLB}

The SLB contained one part of negatively charged phospholipids (DOPS) and four parts of neutral phospholipids (DOPC) because the chosen ratio is similar to the composition of the inner leaflet of neuronal membranes, which is typically enriched with negatively charged phospholipids in the range of $15-30 \mathrm{~mol} \%$. Generally, the interaction of nanoparticles with hydrophilic interfaces requires negative surface charges implying a strong contribution of electrostatic forces during the adsorption event. As shown in Figure 3, the fluorescence intensities of SLB after $16 \mathrm{~h}$ of exposure to a suspension of $\mathrm{ZnO}$ or $\mathrm{TiO}_{2}$ were recorded. The reduced fluorescence intensity of the membrane with $\mathrm{ZnO}(2.5 \mathrm{mg} / \mathrm{ml})$ reflects on the possible $\mathrm{ZnO}$ aggregates deposition on SLB. The measured zeta potential of $\mathrm{ZnO}$ suspension shows the high probability of aggregates formation, which could quench the fluorescence intensity. The possible explanation why we obtained difference with different NP's is because the various (from 3 to 33 $\mathrm{mV})$ zeta potentials lead to stability of suspension $\left(\mathrm{TiO}_{2}\right.$ case) or big aggregates attraction to lipid bilayer ( $\mathrm{ZnO}$ case).

To confirm NP's aggregates attachment to SLB surface, FCS measurements were used (Figure 4) indicating the impact of NP's on the lipids diffusion in the supported lipid bilayer at different concentrations. To investigate the $\mathrm{ZnO}$ and $\mathrm{TiO}_{2} \mathrm{NP}$ 's interaction and aggregation with supported lipid bilayers at different concentrations we employed SAF microscopy combined with the fluorescent correlation spectroscopy (FCS) as a sensitive and efficient method to study the interaction of nanoparticles with supported lipid bilayers under continuous and constant flow conditions. For analysing the FCS data (Figure 4) we used a diffusion model expanded by a second diffusion coefficient in a linear combination $\left(\mathrm{D}, \mathrm{D}_{\mathrm{a}}, \mathrm{D}_{\mathrm{b}}\right)$
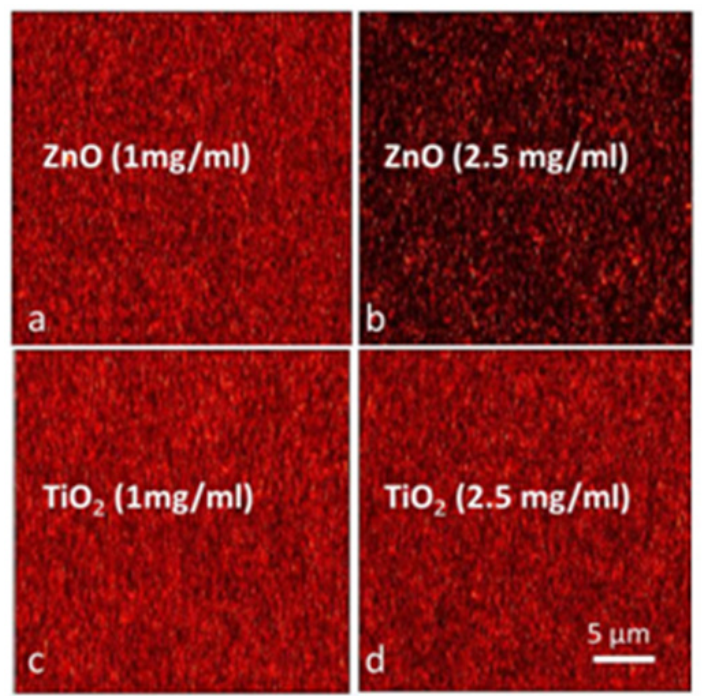

Figure $3 \mathrm{ZnO}$ and $\mathrm{TiO}_{2}$ interaction with lipid membrane. SAF images of the scanned areas acquired after $16 \mathrm{~h}$ of exposure to a suspension of $\mathrm{ZnO}$ (top row) or $\mathrm{TiO}_{2}$ (bottom row) with different concentrations on the lipid membrane bilayer.
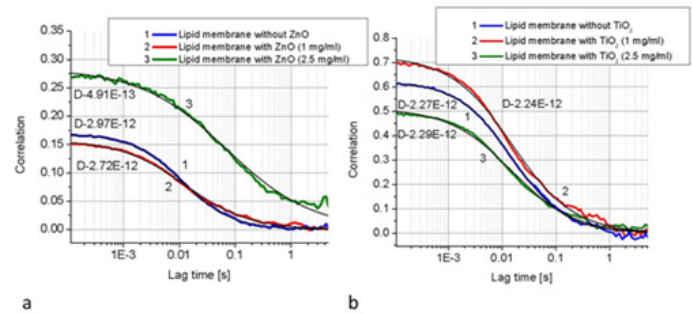

Figure 4 Fluorescence intensities of lipid membrane without (blue line) and with $\mathrm{ZnO}$ (a; red and green lines) or with $\mathrm{TiO}_{2}$ (b; red and green lines). Diffusion curves (from FCS measurements) indicating the difference in the presence (blue) and absence (green) of $2.5 \mathrm{mg} / \mathrm{ml} \mathrm{ZnO}$. The solid black lines represent the fitting curves for corresponding FCS curves.

$$
\begin{aligned}
& G(t)=a^{*} G_{0} *\left(1+4 D_{a} / w_{0}{ }^{2} t\right)^{-1}+b^{*} G_{0} *\left(1+4 D_{b} / w_{0}{ }^{2} t\right)^{-1} \\
& \text { with } \quad D=a * D_{a}+b^{*} D_{b}
\end{aligned}
$$

Where $\mathrm{G}_{0}$ is the intercept of the FCS curve and inverse proportional to the number of the fluorescent molecules and $\omega 0$ is the radius of the 
detection volume. Both NP's show slight variations (D is in range $\left.2-3 \mathrm{E}-12 \mathrm{~m}^{2} \mathrm{~s}^{-1}\right)$ in the diffusion coefficient values, with no defined trend. Although $\mathrm{ZnO}$ with $2.5 \mathrm{mg} / \mathrm{ml}$ concentration has a different impact on lipid diffusion (D-4.91E-13 $\mathrm{m}^{2} \mathrm{~s}^{-1}$ ). The effect could be due to the unstable suspension caused aggregations connections to SLB surface. The influence of addition of the $\mathrm{ZnO}$ or $\mathrm{TiO}_{2} \mathrm{NP}^{2}$ 's to the lipids suspension and NP's effect on the supported lipid membranes was studied by analysing the FCS measurements of the labelled DOPE (diffusion coefficient-D) lipids contained inside membranes before and after nanoparticles addition. Figure 4 illustrates three independent measurements of the diffusion coefficient (D) without addition of nanoparticles and after addition $1 \mathrm{mg} / \mathrm{ml}$ and $2.5 \mathrm{mg} / \mathrm{ml} \mathrm{ZnO}$ or $\mathrm{TiO}_{2}$ concentration, respectively. FCS measurements reveal that the significant reduction of D occurs only after addition of $2.5 \mathrm{mg} / \mathrm{ml}$ $\mathrm{ZnO}$; other samples remained quite stable for a longer period of time.

The significant reduction of $\mathrm{D}$ can be explained as a result of lipid$\mathrm{ZnO}$ aggregates binding. This effect is enlarged with the increasing $\mathrm{ZnO}$ concentration. The higher $\mathrm{ZnO}$ concentration leads to the enhancement of the D reduction. This is in line with other studies, which reported that a noticeable influence over lipid membranes is observed at higher concentrations of cholesterol, proteins or other nanoparticles. ${ }^{26-32}$ This effect is explained by reduction of free area because of tighter lipid packing. To determine if the decrease of the lipids diffusion coefficient is followed by a destruction of the bilayer homogeneity and integrity, scanned SAF images were performed at different times (Figure 3). The scanned images reveal that the bilayer integrity is not altered for 16 hours after nanoparticles addition. Thus, the higher concentration of $\mathrm{ZnO}$ fluorescence intensity is significantly lower from others presented in Figure 3. This let us conclude that the $\mathrm{ZnO}$ addition induces a significant alteration of the mobility of lipids inside the membrane before any aggregation processes of nanoparticles start to occur. The possible explanation is that the $\mathrm{ZnO}$ zeta potential (with $\mathrm{pH} 7.4 \sim+3 \mathrm{mV}$ ) could affect the lipids mobility due to the unstable suspension caused aggregations connections to SLB surface. In opposite, $\mathrm{TiO}_{2}$ zeta potential variation (with $\mathrm{pH} 7.4 \sim 33 \mathrm{mV}$ ) shows stable suspension. Experimental results suggest that the nanoparticlemembrane interaction depends on the NP charge as well as the zeta potential. Cationic nanoparticles are known to penetrate through the cell membrane. ${ }^{1}$ It seems evident that the binding of $\mathrm{TiO}_{2}-$ with the membrane takes place through relatively weak contacts since NP did not immerse deeply in the membrane but floated on top of the bilayer surface, enabling diffusion of the membrane, with no adsorption to the membrane. This is in line with other study, where reported that a noticeable and statistically significant $(p<0,001)$ influence on phospholipid vesicles were observed after incubation of $\mathrm{ZnO}$ (but not $\mathrm{TiO}_{2}$ ) nanoparticles. ${ }^{33}$

This is known that agglomerates larger than $0,2 \mu \mathrm{m}$ were found attached to the membrane. ${ }^{34}$ whereas smaller aggregates and single particles could probably cross the membrane through pores or ion channels. ${ }^{35}$ In addition, aggregates adhere to surfaces may therefore cause effects without even entering cells. The effects of NPs on SLB are important for understanding the potential effects of NPs on biological membranes. Limited biological relevance of the results of experiments with SLB does not mean that these results have no value for understanding effects on biological membranes. It is indicated that $\mathrm{ZnO}$ (but not $\mathrm{TiO}_{2}$ ) can cause the changes of the membrane characteristics if it can get close to the phospholipid bilayer.

\section{Conclusion}

In this study, we investigated the impact of $\mathrm{ZnO}$ and $\mathrm{TiO}_{2}$, being essential constituents of drugs and cosmetics, on the dynamics of lipid molecules in bilayer membranes. Using electrostatically attached NP's of positive charge on negatively charged supported model membranes, we demonstrated that the diffusion mobility of lipids within the $\mathrm{ZnO}$ membrane slowed down significantly, depending on concentration. The weak interaction was found between $\mathrm{TiO}_{2}$ and lipid bilayers. As an explanation we suggest a mechanism of strong interaction between positively charged nanoparticles and the negatively charged lipids, thus forming tight nanoparticles-lipid complexes in the case of $\mathrm{ZnO}$. The finding that diffusion of lipid molecules within the membrane significantly depends on interaction with NP represents a new step in understanding the interactions between NP and cells by demonstrating the non disruptive nature of the NP and lipid bilayer interaction and showing the importance of NP surface properties.

\section{Acknowledgements}

This research is funded by the European Social Fund under the Global Grant measure (Grant No. VP1-3.1-SMM-07-K-03-044).

\section{Conflicts of interest}

None.

\section{References}

1. Heikkilä E, Martinez-Seara H, Gurtovenko AA, et al. Atomistic simulations of anionic Au 144 (SR) 60 nanoparticles interacting with asymmetric model lipid membranes. Biochim Biophys Acta. 2014;1838(11):2852-2860.

2. He XC, Lin M, Li F, et al. Advances in studies of nanoparticlebiomembrane interactions. Nanomedicine. 2015;10(1):121-141.

3. Karlsson HL, Cronholm P, Hedberg Y, et al. Cell membrane damage and protein interaction induced by copper containing nanoparticlesImportance of the metal release process. Toxicology. 2013;313(1):59-69.

4. Adjei IM, Sharma B, Labhasetwar V. Nanoparticles: cellular uptake and cytotoxicity. In Nanomaterial. 2014;811:73-91.

5. Lu B, Smith T, Li R, et al. Nanoparticle-Membrane Interactions Studied with Lipid Bilayer Arrays. Biophys J. 2014;106(2):415a.

6. Sanjay SS, Pandey A, Ankit P, et al. Fabrication of surfactant sensing membrane with $\mathrm{znO}$ nano-composite. Proceedings of the National Academy of Sciences, India Section A: Physical Sciences 2013;83(3):279-285.

7. Sundaram Sanjay S, Yadav RS, Pandey AC. Synthesis of lamellar porous photocatalyticnanozno with the help of anionic surfactant. $A d v$ Mater Lett. 2013;4(5).

8. Asl SK, Sadrnezhaad S. The seeding effect on the microstructure and photocatalytic properties of $\mathrm{znO}$ nano powders. Materials Letters. 2010;64(18):1935-1938.

9. Maurya A, Chauhan P, Mishra A and Pandey AK. Surface functionalization of $\mathrm{TiO}_{2}$ with plant extracts and their combined antimicrobial activities against E. faecalis and E. Coli. J Res Updates Polymer Sci. 2012;1(1):43-51.

10. Šimundić $\mathrm{M}$, Drašler $\mathrm{B}$, Šuštar $\mathrm{V}$, et al. Effect of engineered $\mathrm{TiO}_{2}$ and $\mathrm{ZnO}$ nanoparticles on erythrocytes, platelet-rich plasma and giant unilamelar phospholipid vesicles. BMC vet Res. 2013;9(1):7.

11. Ruckstuhl T, Verdes D. Supercritical angle fluorescence (SAF) Microscopy. Opt Express. 2004;12(18):4246-4254.

12. Verdes D, Ruckstuhl T, Seeger S. Parallel two-channel near- and farfield fluorescence microscopy. J Biomed Opt. 2007;12(3):034012.

13. Richter R, Mukhopadhyay A, Brisson A. Pathways of lipid vesicle deposition on solid surfaces: a combined QCM-D and AFM study. Biophys J. 2003;85(5):3035-3047. 
14. Schwille P, Korlach J, Webb WW. Fluorescence correlation spectroscopy with single-molecule sensitivity on cell and model membranes. Cytometry. 1999;36(3):176-182.

15. Bacia K, Scherfeld D, Kahya N, et al. Fluorescence correlation spectroscopy relates rafts in model and native membranes. Biophys $J$. 2004;87(2):1034-1043.

16. Eggeling C, Ringemann $\mathrm{C}$, Medda $\mathrm{R}$, et al. Direct observation of the nanoscale dynamics of membrane lipids in a living cell. Nature. 2009;457:1159-1121

17. Sezgin E, Schwille P. Fluorescence techniques to study lipid dynamics, Cold Spring Harb perspect Biology. 2011;3(11):a009803.

18. Haustein E, Schwille P. Fluorescence correlation spectroscopy: novel variations of an established technique. Annu Rev Biophys Biomol Struct. 2007;36:151-169.

19. Chiantia S, Ries J, Chwastek G, et al. Role of ceramide in membrane protein organization investigated by combined AFM and FCS. Biochim Biophys Acta. 2008;1778(5):1356-1364.

20. Lingwood D, Ries J, Schwille P, et al. Plasma membranes are poised for activation of raft phase coalescence at physiological temperature. Proc Natl Acad Sci. 2008;105(29):10005-10010.

21. García-Sáez AJ, Schwille P. Stability of lipid domains. FEBS Lett. 2010;584(9):1653-1658.

22. García-Sáez AJ, Carrer DC, Schwille P. Fluorescence correlation spectroscopy for the study of membrane dynamics and organization in giant unilamellar vesicles. Methods Mol Biol. 2010;606:493-508.

23. Smoluchowski M. Handbuch der Electrizität und des Magnetismus (Graetz) (vol. II), Barth, Leipzig, Germany. 1921;pp.366.

24. Delgado AV, González-Caballero F, Hunter RJ, et al. Measuremen and interpretation of electrokinetic phenomena. Pure Appl Chem. 2005;77(10):1753-1805.

25. Kaszuba M, Corbett J, Watson FM, et al. High-concentration zeta potential measurements using light-scattering techniques. Philos Trans A Math Phys Eng Sci. 2010;368(1927):4439-4451.
26. Kahya N, Scherfeld D, Bacia K, et al. Lipid domain formation and dynamics in giant unilamellar vesicles explored by fluorescence correlation spectroscopy. J Struct Biol. 2004;147(1):77-89.

27. Kahya N, Schwille P. How phospholipid-cholesterol interactions modulate lipid lateral diffusion, as revealed by fluorescence correlation spectroscopy. J Fluoresc. 2006;16(5):671-678.

28. Sahoo H, Schwille P. Influence of glycosaminoglycans on lipid dynamics in supported phospholipid bilayers. Soft Matter. 2013;9(14):3859-3865.

29. Bockmann RA, Hac A, Heimburg T, et al. Effect of sodium chloride on a lipid bilayer. Biophys J. 2003;85(3):1647-1655

30. Sum AK, Faller R, de Pablo JJ Molecular simulation study of phospholipid bilayers and insights of the interactions with disaccharides. Biophys J. 2003;85(5):2830-2844.

31. Blondelle SE, Lohner K, Aguilar MI. Lipid-induced conformation and lipid-binding properties of cytolytic and antimicrobial peptides: determination and biological specificity. Biochim Biophys Acta 1462 1999;(12):89-108

32. Macháň R, Hof M. Lipid diffusion in planar membranes investigated by fluorescence correlation spectroscopy. Biochim Biophys Acta Biomembr. 2010;1798(7):1377-1391.

33. Šimundić $\mathrm{M}$, Drašler $\mathrm{B}$, Šuštar $\mathrm{V}$, et al. Effect of engineered $\mathrm{TiO}_{2}$ and $\mathrm{ZnO}$ nanoparticles on erythrocytes, platelet-rich plasma and giant unilamelar phospholipid vesicles. BMC vet Res. 2013;9:7.

34. Rothen-Rutishauser BM, Schurch S, Haenni B, et al. Interaction of fine particles and nanoparticles with red blood cells visualized with advanced microscopic techniques. Environ Sci Technol. 2006;40(14):4353-4359.

35. Porter AE, Muller K, Skepper J, et al. Uptake of C60 by human monocyte macrophages, its localization and implication for toxicity: studied by high resolution electron microscopy and electron tomography. Acta Biomater. 2006;2(4):409-419. 